$$
z=1+\frac{a_{2 n-1}}{\bar{z}}, \quad \bar{z}=1+\frac{a_{2 n}}{z}, \quad n \geqq 1 \text {. }
$$

This gives

$$
\begin{aligned}
a_{2 n-1} & =(z-1) \bar{z} \\
a_{2 n} & =(\bar{z}-1) z=\bar{a}_{2 n-1},
\end{aligned}
$$

and it is easily seen that all $a_{n}$ lie on the boundary of the parabola. The theorem is now completely proved.

\title{
BIBLIOGRAPHY
}

1. J. F. Paydon, Convergence regions and value regions for continued fractions, this Bulletin, abstract 47-11-473.

2. W. T. Scott and H. S. Wall, A convergence theorem for continued fractions, Transactions of this Society, vol. 47 (1940), pp. 155-172.

3. - Value regions for continued fractions, this Bulletin, vol. 47 (1941), pp. $580-585$.

The Rice Institute

\section{A TABLE OF COEFFICIENTS FOR NUMERICAL DIFFERENTIATION}

ARNOLD N. LOWAN, HERBERT E. SALZER AND ABRAHAM HILLMAN ${ }^{1}$

The following table lists the coefficients $A_{m, s}$ for $m=1,2, \cdots, 20$ and $s=m, \cdots, 20$ in Markoff's formula for the $m$ th derivative in terms of advancing differences, namely

$$
\omega^{m} f^{(m)}(x)=\sum_{s=m}^{n-1}(-1)^{m+s} A_{m, s} \Delta^{s} f(x)+(-1)^{m+n} \omega^{n} A_{m, n} f^{(n)}(\xi) .
$$

In this formula $\omega$ is the tabular interval and

$$
A_{m, s}=(-1)^{m+s} m B_{s-m}^{(s)} / s(s-m) !
$$

and $B_{s-m}^{(s)}$ is the $(s-m)$ th Bernoulli number of the $s$ th order.

Presented to the Society, April 4, 1942 under the title Coefficients of differences in the expansion of derivatives in terms of advancing differences; received by the editors March 7, 1942.

${ }^{1}$ The results reported here were obtained in the course of the work done by the Mathematical Tables Project, Work Projects Administration, New York City. 
If a function has been tabulated to sufficiently great accuracy and for some suitable interval of the argument along the real axis, the accompanying table may be used to generate the values of the derivatives which in turn may be employed to generate the values of the function in the complex plane within a region where the function is analytic.

The coefficients were computed from the recurrence formula

$$
s A_{m, s}=(s-1) A_{m, s-1}+m A_{m-1, s-1}
$$

and checked by independent calculations using the identity

$$
x(x+1)(x+2) \cdots(x+s-1) \equiv s ! \sum_{j=1}^{s} A_{j, s} x^{j} / j !
$$

From the identity

$$
\left(x+x^{2} / 2+x^{3} / 3+\cdots\right)^{m} \equiv A_{m, m} x^{m}+A_{m, m+1} x^{m+1}+\cdots
$$

it was discovered that a prime $p$ is not effectively present in the denominator of an $A_{m, s}$ for which $s<m+p-1$. The cancellation of prime factors in accordance with this rule was a further check on the accuracy of the work.

The Markoff formula is used at the beginning and end of a table where advancing differences are the only types available. For a full discussion see L. M. Milne-Thomson, The Calculus of Finite Differences, chap. 7, pp. 157-159. According to Milne-Thomson the relative simplicity of the remainder term is another advantage over central difference formulae.

Comparison of the Markoff coefficients with central difference coefficients shows the latter to be much smaller and obviously more convenient for obtaining the derivatives of a polynomial sufficiently far away from the ends of a table. However for many important functions in applied mathematics such as Bessel, error, and gamma functions, use of the Markoff formula for a polynomial approximation of some fixed degree might yield a smaller total error due to the particular form of its remainder term.

The first few coefficients of the various formulae may be found in H. T. Davis, Table of the Higher Mathematical Functions, vol. 1, pp. 73-77; Whittaker and Robinson, Calculus of Observations, pp. 62-65, and in an article by W. S. Bickley Numerical differentiation near the limits of a difference table, Philosophical Magazine, (7), vol. 33 (1942), pp. 12-14. (This article lists coefficients of the first 12 derivatives up to those of the 12 th difference.) 
Coefficients $A_{m}, \delta$ IN Markoff's Expansion

\begin{tabular}{|c|c|c|c|c|c|c|c|c|c|c|c|c|c|c|}
\hline$m l^{s}$ & 1 & 2 & 3 & 4 & 5 & 6 & 7 & 8 & 9 & 10 & 11 & 12 & 13 & 14 \\
\hline \multirow{2}{*}{1} & \multirow{2}{*}{1} & 1 & 1 & 1 & 1 & 1 & 1 & 1 & 1 & 1 & 1 & 1 & 1 & 1 \\
\hline & & 2 & 3 & $\overline{4}$ & 5 & $\overline{6}$ & $\overline{7}$ & $\overline{8}$ & $\overline{9}$ & 10 & $\overline{11}$ & $\overline{12}$ & 13 & 14 \\
\hline \multirow{2}{*}{2} & & 1 & 1 & 11 & 5 & 137 & 7 & 363 & 761 & 7129 & 671 & 83711 & 6617 & 1145993 \\
\hline & & 1 & 1 & $\overline{12}$ & $\overline{6}$ & $\overline{180}$ & $\overline{10}$ & $\overline{560}$ & $\overline{1260}$ & 12600 & 1260 & 166320 & 13860 & 2522520 \\
\hline \multirow{2}{*}{3} & & & 1 & 3 & 7 & 15 & 29 & 469 & 29531 & 1303 & 16103 & 190553 & 128977 & 9061 \\
\hline & & & 1 & 2 & 4 & 8 & $\overline{15}$ & 240 & 15120 & 672 & 8400 & 100800 & 69300 & 4950 \\
\hline \multirow{2}{*}{4} & & & & & & 17 & 7 & 967 & 89 & 4523 & 7645 & 341747 & 412009 & 9301169 \\
\hline & & & & 1 & 2 & $\overline{6}$ & $\overline{2}$ & $\overrightarrow{240}$ & $\overline{20}$ & $\overline{945}$ & $\overline{1512}$ & 64800 & 75600 & 1663200 \\
\hline \multirow{2}{*}{5} & & & & & 1 & 5 & 25 & 35 & 1069 & 285 & 31063 & 139381 & 1148963 & 355277 \\
\hline & & & & & 1 & $\overline{2}$ & $\overline{6}$ & 6 & $\overline{144}$ & $\overline{32}$ & 3024 & 12096 & $\overline{90720}$ & 25920 \\
\hline \multirow{2}{*}{6} & & & & & & & & 23 & & 3013 & 781 & 242537 & 48035 & 1666393 \\
\hline & & & & & & 1 & 3 & $\overline{4}$ & 9 & $\overline{240}$ & $\overline{48}$ & 12096 & 2016 & 60480 \\
\hline \multirow{2}{*}{7} & & & & & & & & 7 & 91 & 105 & 4781 & 13321 & 314617 & 790153 \\
\hline & & & & & & & 1 & $\overline{2}$ & $\overline{12}$ & $\overline{8}$ & $\overline{240}$ & $\overline{480}$ & $\overline{8640}$ & 17280 \\
\hline \multirow{2}{*}{8} & & & & & & & & 1 & 4 & 29 & 55 & 10831 & 897 & 944311 \\
\hline & & & & & & & & 1 & 4 & $\overline{3}$ & $\overline{3}$ & $\overline{360}$ & $\overline{20}$ & $\overline{15120}$ \\
\hline \multirow{2}{*}{9} & & & & & & & & & 1 & 9 & 12 & 99 & 1747 & 5551 \\
\hline & & & & & & & & & 1 & $\overline{2}$ & 12 & $\overline{4}$ & $\overline{40}$ & $\overline{80}$ \\
\hline \multirow{2}{*}{10} & & & & & & & & & & 1 & 5 & 175 & $\underline{65}$ & 491 \\
\hline & & & & & & & & & & 1 & 3 & $\overline{12}$ & $\overline{2}$ & $\overline{8}$ \\
\hline \multirow{2}{*}{11} & & & & & & & & & & & 1 & 11 & 209 & 1001 \\
\hline & & & & & & & & & & & & $\overline{2}$ & $\overline{12}$ & $\overline{24}$ \\
\hline \multirow{2}{*}{12} & & & & & & & & & & & & 1 & 6 & 41 \\
\hline & & & & & & & & & & & & & 0 & $\overline{2}$ \\
\hline \multirow{2}{*}{13} & & & & & & & & & & & & & 1 & 13 \\
\hline & & & & & & & & & & & & & 1 & $\overline{2}$ \\
\hline 14 & & & & & & & & & & & & & & 1 \\
\hline
\end{tabular}

MATHEMATICAL TABLES PROJECT 70 Columbus Avenue 
$\omega^{m} D^{m} f(x) \sim \sum_{s=m}^{s=n}(-1)^{m+s} A_{m, s} \Delta^{s} f(x)$

\begin{tabular}{|c|c|c|c|c|c|c|}
\hline 15 & 16 & 17 & 18 & 19 & 20 & \\
\hline 1 & 1 & 1 & 1 & 1 & $\overline{1}$ & \\
\hline$\overline{15}$ & $\overline{16}$ & $\overline{17}$ & $\overline{18}$ & $\overline{19}$ & $\overline{20}$ & 1 \\
\hline 1171733 & 1195757 & 143327 & 42142223 & 751279 & 275295799 & 0 \\
\hline$\overline{2702700}$ & $\overline{2882880}$ & $\overline{360360}$ & $\overline{110270160}$ & $\overline{2042040}$ & $\overline{775975200}$ & 2 \\
\hline$\overline{30946717}$ & 39646461 & 58433327 & 344499373 & 784809203 & 169704792667 & \\
\hline$\overline{17199000}$ & $\overline{22422400}$ & $\overline{33633600}$ & $\overline{201801600}$ & $\overline{467812800}$ & $\overline{102918816000}$ & 3 \\
\hline 406841 & $\overline{35118025721}$ & $\overline{4446371981}$ & 80847323107 & 2263547729 & 32262100943 & \\
\hline 71280 & 6054048000 & 756756000 & $\overline{13621608000}$ & 378378000 & 5360355000 & 4 \\
\hline 21939781 & 2065639 & 2195261857 & 371446039969 & 27566944753 & 31938836201 & 5 \\
\hline$\overline{1496880}$ & 133056 & $\overline{134534400}$ & $\overline{21794572800}$ & $\overline{1556755200}$ & $\overline{1743565824}$ & 5 \\
\hline 22463 & 277382447 & 38101097 & 1356664151597 & 162356544377 & 694142313941 & 6 \\
\hline 720 & 7983360 & 997920 & 32691859200 & 3632428800 & $\overline{14529715200}$ & 0 \\
\hline 899683 & 2271089 & 86853967 & 13195009 & 227663026369 & 2022480780283 & \\
\hline$\overline{16200}$ & $\overline{34560}$ & $\overline{1140480}$ & $\overline{152064}$ & 2335132800 & 18681062400 & 7 \\
\hline 35717 & 54576553 & 8424673 & 334947281 & 9764119 & 5013017410969 & 0 \\
\hline$\overline{432}$ & 518400 & 64800 & 2138400 & 52800 & 23351328000 & $\gamma$ \\
\hline 515261 & 23915 & 76492463 & 21878439 & 4065163957 & 3975325483 & 0 \\
\hline$\overline{5040}$ & 168 & 403200 & 89600 & 13305600 & 10644480 & 9 \\
\hline 2485 & 324509 & 59279 & 79243781 & 11795941 & 6063698587 & \\
\hline$\overline{24}$ & 2016 & 252 & $\overline{241920}$ & 26880 & 10644480 & 10 \\
\hline 30217 & 1199 & 494351 & 1513391 & 18843187 & 367394203 & 11 \\
\hline$\overline{360}$ & $\overline{8}$ & 2016 & 4032 & 34560 & $\overline{483840}$ & 11 \\
\hline 105 & 26921 & 6341 & 5490071 & 976163 & 354467473 & \\
\hline$\overline{2}$ & $\overline{240}$ & $\overline{30}$ & $\overline{15120}$ & $\overline{1680}$ & $\overline{403200}$ & 12 \\
\hline$\frac{143}{6}$ & 65 & $\frac{35269}{240}$ & $\frac{46631}{160}$ & $\frac{3965533}{7560}$ & $\frac{10596053}{12096}$ & 13 \\
\hline 7 & $\frac{329}{12}$ & $\frac{238}{3}$ & $\frac{136241}{720}$ & $\frac{31521}{80}$ & $\frac{6406481}{8640}$ & 14 \\
\hline
\end{tabular}

WORK PROJECTS ADMINISTRATION

New York City 
A. N. LOWAN, H. E. SALZER AND ABRAHAM HILLMAN

Coefficients $A_{m}, s$ in Markoff's Expansion $\omega_{m} D^{m} f(x) \sim \sum_{s=m}^{s=n}(-1)^{m+s} A_{m, s} \Delta^{s} f(x)$

\begin{tabular}{|c|c|c|c|c|c|c|}
\hline$m$ & 15 & 16 & 17 & 18 & 19 & 20 \\
\hline 15 & 1 & $\frac{15}{2}$ & $\frac{125}{4}$ & $\frac{765}{8}$ & $\frac{11519}{48}$ & $\frac{50255}{96}$ \\
\hline 16 & & 1 & 8 & $\frac{106}{3}$ & 114 & $\frac{18017}{60}$ \\
\hline 17 & & & 1 & $\frac{17}{2}$ & $\frac{119}{3}$ & $\frac{1615}{12}$ \\
\hline 18 & & & & 1 & 9 & $\frac{117}{4}$ \\
\hline 19 & & & & & 1 & $\frac{19}{2}$ \\
\hline 20 & & & & & & 1 \\
\hline
\end{tabular}

New York City 